\title{
Balancing scientific tensions
}

\section{To the Editor - Within}

nanoecotoxicology, various scientific tensions exist in dynamic interplay. For example, exploratory and curiositydriven basic research competes with strategic-, applied- and regulatoryoriented approaches; research done within a paradigm of environmental chemistry must rival with a biological one; and research focused on well-controlled experimental set-ups vies with that prioritizing testing under complex and variable real-world conditions. All these research orientations are valuable, and just as in the concept of yin and yang, they should be regarded as complementary forces between which it is important to find a balance.

Disconcertingly, however, the urgent need for environmental, health and safety data to support regulatory decision-making has created pressure to develop and use international standards in nanoecotoxicology, which can potentially cause an imbalance in the research orientations. Standardization may be necessary for reliability, comparability and regulatory relevance of nanoecotoxicological studies, but we should recognize and address the three double-binds that this creates.

A 'double-bind' is a persistent type of dilemma whereby two choices are in tension, and success in one inevitably creates problems in the other. The first double-bind is a temporal one - it being arguably both too early and too late for standardization of nanoecotoxicology testing. Standardization is overdue because nano-products are already commercially available and data generated using existing methods may not be appropriate. It is too early, however, because results from initial testing are too diverse to offer any clear patterns on which the standardization of testing methods can be based. Even small modifications in sample preparation, composition of the test media or instrumentation can greatly influence the results of nanoparticle testing, making it difficult to condense current knowledge into ideal protocols.

The second double-bind relates to the tension between pursuing tests under real environmental conditions and the need for well-controlled experimental set-ups. The Organisation for Economic Corporation and Development (OECD) spearheaded the development of testing guidelines for chemicals to ensure mutual cross-national acceptance of data that are relevant for classification, labelling and hazard assessment. However, this commitment to standardize experimental conditions tends to undermine the importance of testing under the complex and varied environmental conditions into which nanomaterials are released.

The third double-bind concerns how pursuing knowledge according to any one particular approach inevitably creates selective ignorance, leading researchers to only partially understand complex phenomena. This does not imply that we are better off not pursuing knowledge at all, but rather highlights the importance of being aware of how paradigms shape and delimit what knowledge is produced. Given the complexity of nanoparticle properties, experimental media and other influencing factors, at present there is no single paradigm or testing approach that can be claimed to outrank others. Under such high uncertainty, it is important to actively reflect on the assumptions and limitations for all approaches.

The triple double-bind situation facing nanoecotoxicology standardization can seem paralysing. Although there may be no ultimate escape, one way to move forward in a responsible manner is to acknowledge, actively cultivate and support the diverse research areas in nanoecotoxicology as an essential condition for scientific robustness.

Funding bodies, researchers, regulators, publishers and standardization organizations alike need to acknowledge the triple double-bind situation. Funders should ensure that there are opportunities for diverse research approaches to be pursued. Researchers should continue to trial, develop and report on different test methods and communicate the limitations of their knowledge transparently. Scientific journals should remain open towards publishing different types of research (even when consensus seems to be emerging in favour of standardized testing), as well as publishing negative results. Regulators should resist the assumption that public authority requires scientific certainty and should be nuanced in their evaluation of data quality - focusing on both whether a study follows a particular standard and whether it is properly described, well performed and uses well-characterized nanoparticles. Finally, standardization organizations should resist the urge to develop premature standards and remain open, flexible and responsive to ongoing exploratory research and testing.

It is now clear that standards for chemical testing are not appropriate for nanoparticles. However, rushing to develop harmonized standards for nanoecotoxicology and ignoring the three double-binds we face may unwittingly cause undue imbalance in the field. This could create false negatives that would place human and environmental health and safety in danger, and threaten the scientific and public legitimacy of nanoecotoxicology research.

Fern Wickson ${ }^{1 \star}$, Nanna B. Hartmann ${ }^{2}$, Rune Hjorth², Steffen Foss Hansen², Brian Wynne ${ }^{3}$ and Anders Baun ${ }^{2}$ 'Society, Ecology and Ethics Department, Gen $\varnothing \mathrm{k}$ Centre for Biosafety, Forskningsparken PB 6418, 9294 Tromsø, Norway, ${ }^{2}$ Department of Environmental Engineering, Technical University of Denmark, Miljoevej, Building 113, 2800 Kgs. Lyngby, Denmark, ${ }^{3}$ Department of Sociology, Bowland North, Lancaster University, Lancaster LA1 4YT, UK. *e-mail: fern.wickson@genok.no 